\title{
Impact of Mobility on Cooperative Communication
}

\author{
Krishna Srikanth Gomadam and Syed Ali Jafar \\ Electrical Engineering and Computer Science \\ University of California, Irvine, CA 92697-2625 \\ Email:kgomadam@uci.edu,syed@uci.edu
}

\begin{abstract}
We investigate the performance degradation due to rapidly time varying channels in a repetition based coherent cooperative system. We demonstrate that mobility of source affects the performance much more than the mobility of destination, for both amplify and forward (AF) and demodulate and forward (DF) relays, despite the symmetry of the network. Exploiting the property of FSK modulation that allow us to detect either coherently or noncoherently or even semi-coherently, we develop ML detection rules for a variety of mobile scenarios. The detection rules that take into account the mobility of the nodes, are mostly hybrids of partially coherent detectors and noncoherent detectors. The performance of these detectors is better than the best of coherent and noncoherent detectors in fast fading and a gain of about $2 \mathrm{~dB}$ is obtained over a wide range of SNR and a gain of almost $3 \mathrm{~dB}$ is achieved at the crossing of coherent and noncoherent curves. As energy efficiency is one of the main objectives for pursuing cooperation and relaying, these hybrid detectors assume significance in fast fading scenarios.
\end{abstract}

\section{INTRODUCTION}

Despite its impressive advantages, employing multiple antennas for transmission and reception is still unattractive due to size and cost limitations of terminals in cellular, ad-hoc and sensor networks. For such systems, space diversity can be achieved by allowing multiple users to cooperate and effectively share their antennas. Space diversity obtained through this form is referred to as cooperative diversity [1] since the terminals share their antennas and other resources to form a virtual antenna array.

Cooperative diversity can be accomplished in a variety of ways. For example, a repetition based cooperation in [2], [3], a space time code based cooperation as in [4], [5] and a channel code based cooperation as in [6], can all achieve full diversity [7], i.e a diversity order equal to the number of paths between the source and the destination. Repetition based cooperative protocols which incur very little complexity at the receiver are spectrally inefficient since each relay requires a separate orthogonal channel for repeating the information [5]. Space-time coded cooperative diversity protocols improve the spectral efficiency of the system as the relays can transmit simultaneously in the same sub-channel. However it increases the decoding complexity at the terminals.

Wireless sensor networks where many nodes cooperate to detect an event and forward the information, need energy efficient relaying strategies, to prolong the network lifetime. Certain applications of wireless sensor networks require the system to operate in a highly time selective environment. Thus a choice must be made between coherent and noncoherent modulation/detection. For a coherent scheme to operate reliably in a fast fading environment, the channel has to be estimated quite frequently. And in a pilot symbol assisted modulation, this leads to both spectral and energy inefficiency.
A constraint on the training rate would result in severe performance degradation leading to an error floor. Therefore, noncoherent schemes are preferred although they bring in a 3 $\mathrm{dB}$ performance penalty compared to ideal coherent detection. In general, the channel can be broadly modeled as $h=h_{c}+\Delta h$ where $h_{c}$ is the estimate of the channel obtained through training and $\Delta h$ denotes the estimation error. Most receivers ignore $\Delta h$ when operating coherently and similarly $h_{c}$ is not considered during noncoherent detection. However there is a scope for performance improvement if both $h_{c}$ and the statistics of $\Delta h$ are included in the detection process. With some modulation schemes like FSK, a combination of coherent and noncoherent detection [8], [9] can be performed utilizing partial channel state, resulting in a significant energy savings. Partial channel state here refers to the channel information acquired via training at a very low rate, that gets outdated due to rapid channel variations.

In a relay network, mobility of a few nodes need not make the entire set of channels time selective. With appropriate detection strategies that utilize partial CSI, performance improvement can be obtained over a complete noncoherent detection. In Section V, we propose a variety of partially coherent detectors for a repetition based cooperative network, for both $\mathrm{AF}$ and DF relays. The detection rules obtained take account of the mobility scenario and various CSI assumptions at the relay and the destination. For cooperation with a DF relay, we follow the framework in [10], so that the detection rules can be implemented using the piecewise linear approximations. Numerical results are provided to substantiate the merits of the detectors. As energy efficiency is one of the main objectives for pursuing cooperation and relaying, these hybrid detectors assume significance in fast fading scenarios.

\section{SYSTEM MODEL}

Our model consists of a source-destination pair with a single relay as shown in Fig. 1. A TDMA protocol [4] based on half duplex operation, is chosen in which the source $S$ transmits to both the relay $R$ and destination $D$ in the first slot. In the second slot the relay transmits to the destination, while the source remains idle. The set of equations given below summarize the operations taking place for the $k^{t h}$ symbol.

$$
\begin{gathered}
y(k)=h(k) x(k)+n_{D 1}(k) \\
r(k)=g(k) x(k)+n_{R}(k) \\
z(k)=f(k) x_{r}(k)+n_{D 2}(k)
\end{gathered}
$$

At both the relay and the destination, the received signal is corrupted by additive white Gaussian noise with variance $N_{0}$. For amplify and forward (AF) relaying, $x_{r}(k)=\beta_{k} r_{k}$. The relay amplification factor, $\beta_{k}$ is chosen to satisfy an 


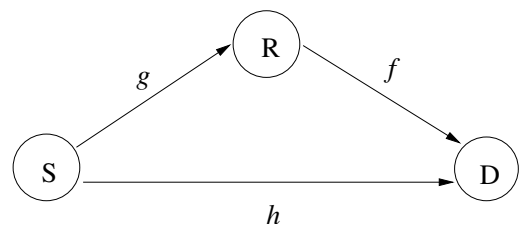

Fig. 1. System Model

average power constraint. For a demodulate and forward relay, $x_{r}(k)=\hat{x}(k)$, where $\hat{x}(k)$ is obtained after demodulating $r(k)$ followed by modulation for transmission to the destination.

We assume all the channels to be frequency nonselective but time selective, modeled by an autoregressive (AR) process. It is indicated in [11] that the first order AR model is a good approximation to the actual fading process.

$$
\begin{aligned}
& h(k)=a_{1} h(k-1)+\sqrt{1-a_{1}^{2}} \Delta h(k) \\
& g(k)=a_{2} g(k-1)+\sqrt{1-a_{2}^{2}} \Delta g(k) \\
& f(k)=a_{3} f(k-1)+\sqrt{1-a_{3}^{2}} \Delta f(k)
\end{aligned}
$$

where $0<a_{1}, a_{2}, a_{3} \leq 1$ indicates the channel variation rate. $\Delta h(k), \Delta g(k)$ and $\Delta f(k)$, the varying component of the channels are independent and identically distributed (iid), zero mean complex Gaussian random processes with variances $\sigma_{1}^{2}, \sigma_{2}^{2}$ and $\sigma_{3}^{2}$ respectively.

It can be noticed from the above equations (2) that the lower the value of $a_{i}$ (i=1,2 and 3 representing each of the three links), the greater is the channel variation rate. The channel realizations become iid when $a_{i}=0$ while $a_{i}=1$ models quasi-static fading. The relationship between the Doppler frequency and $a$ can be approximated using Jakes autocorrelation model [12], given by

$$
a_{i}=\mathcal{J}_{0}\left(2 \pi f_{D_{i}} T_{s}\right),
$$

where $\mathcal{J}_{0}(x)$ is the zeroth order Bessel function of the first kind, $f_{D_{i}}=\frac{f_{c} v_{i}}{c}=\frac{v_{i}}{\lambda}$ is the Doppler shift and $T_{s}$ is the symbol duration. We assume that both the relay and the destination obtain accurate estimate of their channels once every $N$ symbols, via training. The channel estimation rate is restricted to be the same for all the links even though the channel variation rate might be different for each of the links and in general need not completely track the channel. With this definition, knowledge of the S-D link $h(k)$ and R-D link $f(k)$ at the destination can be described as a complex Gaussian random process,

$$
\begin{aligned}
\widehat{h}_{k} & \sim \mathcal{C N}\left(a_{1}^{k} h_{0}, 1-a_{1}^{2 k}\right) \\
\widehat{f}_{k} & \sim \mathcal{C N}\left(a_{3}^{k} f_{0},\left(1-a_{3}^{2 k}\right) \sigma_{1}^{2}\right), \quad k=1,2, \ldots, N .
\end{aligned}
$$

Similarly the knowledge of the S-R link, $g(k)$, at the relay, can be described by $\widehat{g}_{k} \backsim \mathcal{C N}\left(a_{2}^{k} g_{0},\left(1-a_{2}^{2 k}\right) \sigma_{2}^{2}\right)$.

\section{A. Relay Amplification Factor in a time varying scenario}

The relay amplification factor is constrained to satisfy an average energy constraint, $\mathcal{E}\left(\left|\beta_{k} r_{k}\right|^{2}\right)=E_{s}$. When instantaneous CSI is available, the relay amplification factor $\beta(k)$ is chosen to satisfy the energy constraint for each realization of the channel $g(k)$.

$$
\beta_{k}^{2}=\frac{E_{s}}{|g(k)|^{2} E_{s}+N_{0}}
$$

However it is hard to obtain instantaneous CSI in a rapidly time varying channel. In this context, the channel information obtained via training gets outdated soon and can only provide partial information. Using this partial knowledge for calculating the amplification factor for the $k^{t h}$ symbol duration, we have

$$
\begin{aligned}
\beta_{k}^{2} & =\frac{E_{s}}{\mathcal{E}\left(|g(k)|^{2}\right) E_{s}+N_{0}} \\
& =\frac{E_{s}}{\left(a_{2}^{2 k}\left|g_{0}\right|^{2}+\left(1-a_{2}^{2 k}\right) \sigma_{g}^{2}\right) E_{s}+N_{0}} .
\end{aligned}
$$

When absolutely no CSI is available,

$$
\beta_{k}^{2}=\frac{E_{s}}{\sigma_{g}^{2} E_{s}+N_{0}},
$$

is an optimal choice. It can be readily seen that the relay gains for the coherent and noncoherent case are the special forms of (6), obtained by setting $a=1$ and $a=0$ respectively. As the quality of the channel estimate degrades with symbol position, the relay gain also exhibits more noncoherent behavior with increase in $k$. It is important to note that the performance of an AF cooperative system is affected by the choice of the relay amplification factor [13].

In the next section, we first highlight the problems associated with a pure coherent detection in fast fading environments for both DF and AF systems. The analysis is then carried out for FSK modulation in which a partially coherent detection leads to simultaneous coherent and noncoherent detection resulting in an optimal performance in time varying channels.

\section{AMPLIFY AND FORWARD WITH NON IDEAL COHERENT PSK DETECTION}

Let the symbol transmitted at the $k^{t h}$ symbol duration be

$$
x_{k}=\sqrt{E_{s}} e^{j \phi_{k}},
$$

where $\phi_{k} \in[\pi,-\pi]$, the BPSK constellation set and $E_{s}$ is the symbol energy. Although the optimal decoding would be to perform sequence estimation [14] for a channel with memory, the complexity associated with it is too prohibitive to be implemented in simple receivers as in sensor and ad-hoc networks. We therefore use conventional symbol by symbol processing as the detection strategy at the receivers.

\section{A. Maximum Likelihood Detection}

We assume that the destination has knowledge of all the channels $h_{0}, g_{0}$ and $f_{0}$ estimated during the start of the frame and also the channel statistics $a_{1}, a_{2}$ and $a_{3}$ which are related to the mobility of the nodes. The joint density of the received symbols $y(k)$ and $z(k)$ conditioned on the input symbol and the partial CSI is given by

$$
\begin{array}{r}
\operatorname{Pr}\left(y(k), z(k) \mid x_{k}, a_{1}, a_{2}, a_{3}, h_{0}, f_{0}, g_{0}\right)=\operatorname{Pr}\left(y(k) \mid x_{k}, a 1, h_{0}\right) . \\
\operatorname{Pr}\left(z(k) \mid x_{k}, a_{2}, a_{3}, g_{0}, f_{0}\right) . \\
\operatorname{Pr}\left(y(k) \mid x_{k}, a_{1}, h_{0}\right)=\mathcal{C N}\left(y(k): \sqrt{E_{s}} a_{1}^{2 k} h_{0}, V_{1}(k)\right) \\
\operatorname{Pr}\left(z(k) \mid x_{k}, a_{2}, a_{3}, g_{0}, f_{0}\right)=\mathcal{C N}\left(z(k): \sqrt{E_{s}} \beta_{k} a_{2}^{k} a_{3}^{k} g_{0} f_{0}, V_{2}(k)\right)
\end{array}
$$


Here we do not consider the non-Gaussian noise term $\left(\sqrt{1-a_{2}^{2 k}} \Delta g+n_{R}\right) n_{D_{2}}(k)$, which is negligible, as it is done in the analysis of differential PSK in [8], [15].

$$
\begin{gathered}
V_{1}(k)=\left(1-a_{1}^{2 k}\right) E_{s}+N_{0} \\
V_{2}(k)=\beta_{k}^{2} E_{s}\left(a_{3}^{2 k}\left|f_{0}\right|^{2}\left(1-a_{2}^{2 k}\right)\left(1-a_{3}^{2 k}\right) a_{2}^{2 k}\left|g_{0}\right|^{2}\right)+ \\
a_{3}^{2 k}\left|f_{0}\right|^{2} \beta_{k}^{2} N_{0}+N_{0}
\end{gathered}
$$

The optimal detection involves maximal ratio combining of the received symbols scaled by their variances.

$$
\widehat{d_{k}}=a_{1}^{k} h_{0}^{*} \sqrt{E_{s}} \frac{y(k)}{V_{1}(k)}+\beta(k) a_{2}^{k} a_{3}^{k} g_{0}^{*} f_{0}^{*} E_{s} \frac{z(k)}{V_{2}(k)}
$$

The instantaneous SNR $\gamma_{k}$ is then given by

$$
\begin{aligned}
\gamma_{k}= & \frac{a_{1}^{2 k}\left|h_{0}\right|^{2} E_{s}}{\left(1-a_{1}^{2 k}\right)+N_{0}}+ \\
& \frac{a_{2}^{2 k} a_{3}^{2 k}\left|g_{0}\right|^{2}\left|f_{0}\right|^{2} E_{s}}{a_{3}^{2 k}\left|f_{0}\right|^{2}\left(1-a_{2}^{2 k}+N_{0}\right)+a_{2}^{2 k}\left(1-a_{3}^{2 k}\right)\left|g_{0}\right|^{2}+\frac{N_{0}}{E_{s} \beta^{2}}} .
\end{aligned}
$$

In order to obtain the probability of error or average SNR, the above equation has to be integrated over the channel gains. However the integral is intractable and results in incomplete gamma functions. This problem is also encountered in [3] where a closed form expression for the symbol error probability is obtained with high SNR approximations. Building upon methods in [16], it was shown in [3] that

$$
\overline{P_{e}} \approx \frac{3}{4 K^{2}} \frac{1}{\Gamma_{h}}\left(\frac{1}{\Gamma_{g}}+\frac{1}{\Gamma_{f}}\right),
$$

where $\mathrm{K}$ is a constant dependent on the modulation scheme and $\Gamma_{h}, \Gamma_{f}$ and $\Gamma_{k}$ are the average SNR of the links $h, g$ and $f$ respectively. Employing (9) to find the average probability of error for each symbol and then averaging it over the entire block of $N$ symbols, we arrive at the average probability of error given by

$$
\overline{P_{e}} \approx \frac{3}{16}\left[T_{2}+T_{3}\right]
$$

where

$$
\begin{aligned}
T_{i}=\frac{\left(1+\frac{N_{0}}{E_{s}}\right)\left(1+\frac{N_{0}}{\sigma_{i}^{2} E_{s}}\right)}{N}\left(\frac{\frac{1}{\left(a_{1} a_{i}\right)^{2 N}}-1}{\frac{1}{\left(a_{1} a_{i}\right)^{2}}-1}\right)-\frac{1+\frac{N_{0}}{E_{s}}}{N}\left(\frac{\frac{1}{a_{i}^{2 N}}-1}{\frac{1}{a_{i}^{2}}-1}\right) \\
-\frac{1+\frac{N_{0}}{\sigma_{i}^{2} E_{s}}}{N}\left(\frac{\frac{1}{a_{1}^{2 N}}-1}{\frac{1}{a_{1}^{2}}-1}\right)+1, \quad i=2,3
\end{aligned}
$$

This expression is accurate at high SNR and the error floor caused due to constrained channel estimation rate can be found.

$$
\begin{gathered}
\overline{P_{e}}=\frac{3}{16 N}\left\{\left(\frac{\frac{1}{\left(a_{1} a_{2}\right)^{2 N}}-1}{\left(a_{1} a_{2}\right)^{2}}+\frac{\frac{1}{\left(a_{1} a_{3}\right)^{2 N}}-1}{\left.\bar{a}_{1} a_{3}\right)^{-1}}\right)-\left(\frac{\frac{1}{a_{2}^{2 N}-1}}{\frac{2}{a_{2}^{2}}-1}+\frac{\frac{1}{a_{3}^{2 N}}-1}{\frac{1}{a_{3}^{2}}-1}\right)\right\} \\
-\frac{3}{8 N}\left(\frac{\frac{1}{a_{1}^{2 N}}-1}{\frac{1}{a_{1}^{2}}-1}-N\right)
\end{gathered}
$$

\section{B. Source Mobility versus Destination Mobility}

Suppose there are two cooperative scenarios with a static relay in which either the source or the destination is mobile but not both. Naturally both the scenarios are expected to perform identically due to the symmetry. However source mobility affects the performance slightly more than destination mobility in a coherent cooperative system, regardless of whether the relay scaling factor depends on partial channel knowledge or not. When $\beta$ does not depend on partial knowledge of

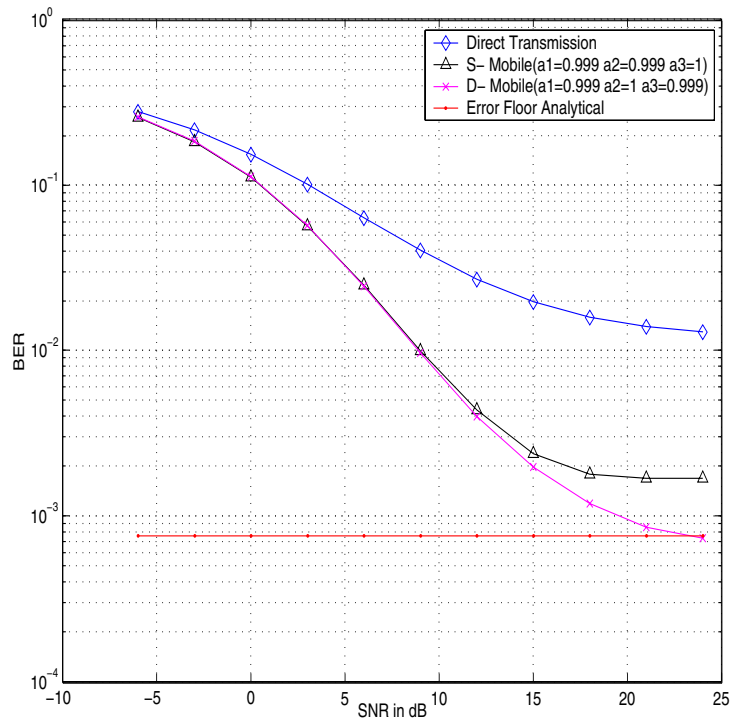

Fig. 2. BER performance of the cooperative $\mathrm{AF}$ schemes at $\mathrm{N}=50$.

$S-R$ link, it is readily seen that $V_{2}(k)$ is greater when $\left(a_{2}, a_{3}\right)=(a, 1)$ than when $\left(a_{2}, a_{3}\right)=(1, a)$.

When equation (6) is used to determine $\beta$, the variance of the relayed path for both the cases is given by

$$
\begin{gathered}
V_{2}^{\mathrm{S}}(k)=\frac{\left(\left(1-a^{2 k}\right) \sigma_{g}^{2}+N_{0}\right)\left|f_{0}\right|^{2}}{a^{2 k}\left|g_{0}\right|^{2}+\left(1-a^{2 k}\right) \sigma_{g}^{2}}+N_{0} \\
V_{2}^{\mathrm{D}}(k)=\frac{\left(1-a^{2 k}\right) \sigma_{f}^{2}\left|g_{0}\right|^{2}}{\left|g_{0}\right|^{2}}+a^{2 k}\left|f_{0}\right|^{2} N_{0}+N_{0} .
\end{gathered}
$$

In the above expressions note that $E_{s}=1$ and there is no loss of generality. At high SNR, we have

$$
\begin{gathered}
V_{2}^{\mathrm{S}}(k)=\frac{\left(1-a^{2 k}\right) \sigma_{g}^{2}\left|f_{0}\right|^{2}}{a^{2 k}\left|g_{0}\right|^{2}+\left(1-a^{2 k}\right) \sigma_{g}^{2}} \\
V_{2}^{\mathrm{D}}(k)=\frac{\left(1-a^{2 k}\right) \sigma_{f}^{2}\left|g_{0}\right|^{2}}{\left|g_{0}\right|^{2}}=\left(1-a^{2 k}\right) \sigma_{f}^{2} \\
\mathcal{E}\left[V_{2}^{\mathrm{S}}(k)\right]=\sigma_{g}^{2}\left(1-a^{2 k}\right)\left(\mathcal{E}_{f_{0}}\left[\left|f_{0}\right|^{2}\right] \mathcal{E}_{g_{0}}\left[\frac{1}{a^{2 k}\left|g_{0}\right|^{2}+\left(1-a^{2 k}\right) \sigma_{g}^{2}}\right]\right) \\
>\quad\left(1-a^{2 k}\right) \sigma_{g}^{2}=V_{2}^{D}(k)
\end{gathered}
$$

Fig. 2 compares the performance of direct transmission and coherent AF system with a static relay. Both the cases with source mobile and destination mobile are plotted. The relay employs a scaling as mentioned in (6). Difference in the performance between the source mobility and the destination mobility scenarios can be observed. Consider the case where the relay alone is mobile. As the direct path is time invariant, the relay mobility case performs better than source or destination mobility. This can also be observed in the error probability expression in (10). In this case, however it should be noted that the gain achieved from cooperation (over direct transmission) is less.

\section{Demodulate And Forward}

The relay demodulates its received symbol $r(k)$ and modulates it again for transmitting it with its own power constraint. As there is a possibility of decision error at the relay, the 
detector at the destination must take account of the error propagation at the relay. The joint density of the received symbols $y(k)$ and $z(k)$ is

$$
\begin{array}{r}
\operatorname{Pr}\left(y(k), z(k) \mid x_{k}, a 1, a 2, a 3, h_{0}, f_{0}, g_{0}\right)=\operatorname{Pr}\left(y(k) \mid x_{k}, a 1, h_{0}\right) . \\
\operatorname{Pr}\left(z(k) \mid x_{k}, a 2, a 3, g_{0}, f_{0}\right) .
\end{array}
$$

For BPSK modulation, error occurs at the relay when $x(k)$ is detected as $-x(k)$. Including the decision error at the relay in the detection rule,

$$
\begin{array}{r}
\operatorname{Pr}\left(z(k) \mid x(k), a 2, a 3, f_{0}\right)=(1-\epsilon(k)) \operatorname{Pr}\left(z(k) \mid x_{r}(k)=x(k), a_{3}, f_{0}\right) \\
+\epsilon(k) \operatorname{Pr}\left(z(k) \mid x_{r}(k)=-x(k), a 3, f_{0}\right) .
\end{array}
$$

The average probability of error at the relay for BPSK modulation [8] for $k^{t h}$ symbol position is

$$
\epsilon(k)=\frac{1}{2}\left(1-a_{2}^{2 k} \sqrt{\frac{\sigma_{h}^{2} E_{s}}{\sigma_{h}^{2} E_{s}+N_{0}}}\right) .
$$

$$
\operatorname{Pr}\left(z(k) \mid x(k), a 2, a 3, f_{0}\right)=\frac{1}{\pi\left(\left(1-a_{3}^{2 k}\right) E_{s}+N_{0}\right)} .
$$

$\epsilon(k) \exp \left(-\frac{\left|z(k)+a_{3}^{k} f_{0} \sqrt{E_{s}}\right|^{2}}{\left(1-a_{3}^{2 k}\right) E_{s}+N_{0}}\right)+(1-\epsilon(k)) \exp \left(-\frac{\left|z(k)-a_{3}^{k} f_{0} \sqrt{E_{s}}\right|^{2}}{\left(1-a_{3}^{2 k}\right) E_{s}+N_{0}}\right)$

It is quite straightforward to arrive at the following decision rule.

$$
\begin{aligned}
\hat{d}_{k}= & \frac{4 a_{1}^{k} \sqrt{E_{s}} \mathfrak{R e}\left(h_{0}^{*} y_{1}(k)\right)}{\left(1-a_{1}^{2 k}\right) E_{s}+N_{0}}+ \\
& \ln \left(\frac{\frac{\epsilon(k)}{1-\epsilon(k)}+\exp \left(\frac{4 a_{3}^{k} \sqrt{E_{s}} \mathfrak{R e}\left(f_{0}^{*} y_{2}(k)\right)}{\left(1-a_{3}^{2 k}\right) E_{s}+N_{0}}\right)}{1+\frac{\epsilon(k)}{1-\epsilon(k)} \exp \left(\frac{4 a_{3}^{k} \sqrt{E_{s} \Re_{e}\left(f_{0}^{*} y_{2}(k)\right)}}{\left(1-a_{3}^{2 k}\right) E_{s}+N_{0}}\right)}\right) \lessgtr_{1}^{0} 0
\end{aligned}
$$

The cutoff points, which determine the maximum contribution of the relayed transmission in the decision rule are

$$
T_{k}= \pm \ln \left(\frac{\epsilon(k)}{1-\epsilon(k)}\right)= \pm \ln \left(\frac{1-a_{2}^{k} \sqrt{\frac{E_{s}}{E_{s}+N_{0}}}}{1+a_{2}^{k} \sqrt{\frac{E_{s}}{E_{s}+N_{0}}}}\right)
$$

As it can be seen, an increased probability of error at the relay results in a decreased weight for the relayed transmission, limiting the gain that can be achieved from cooperation. In such a situation, either the channel has to be estimated before $T_{k}$ decreases below a certain threshold or the cooperative mode must be halted. In a mobile source scenario, the maximum contribution from the relayed transmission is $\ln \left(\frac{1+a^{k}}{1-a^{k}}\right)$, whereas when the destination alone is mobile, the contribution is $\frac{4 a^{k} \sqrt{E_{s}} \Re \mathfrak{R}\left(f_{0}^{*} y_{2}(k)\right)}{\left(1-a^{2 k} E_{s}\right.}$, which is not constrained as long as $a \neq 0$. Fig. 3 compares the performance of direct transmission and coherent DF system with a static relay. It can be seen that the performance difference between the destination mobile case and the source mobile case with a DF relay is much more than that with an AF relay.

\section{Partially Coherent Cooperation with FSK MODULATION}

Employing purely coherent detection in a fast fading environment in systems that have a constraint on channel estimation rate clearly results in a serious performance degradation as highlighted in the previous section. Usually noncoherent detection that obviates the need for channel estimation is preferred. However as discussed earlier, noncoherent detection

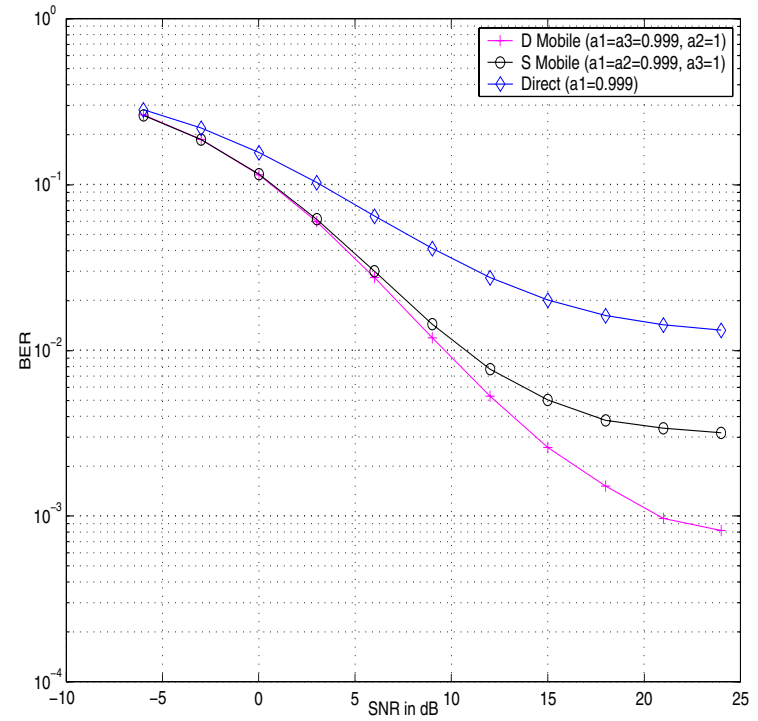

Fig. 3. BER performance of the cooperative DF schemes at $\mathrm{N}=50$.

brings in a $3 \mathrm{~dB}$ loss which may not be acceptable to systems that are keen on saving energy. This performance penalty due to noncoherent detection can be reduced by exploiting partial channel knowledge.

In a fast fading channel, the channel estimate obtained through training gets outdated so quickly that coherent detection cannot be performed. Nevertheless the outdated channel information can still be utilized in the detection process if it would result in a considerable performance improvement. The channel information in this context (4) has both amplitude and phase uncertainties. A detector proposed in [8] exploits this channel information to provide performance improvements over the best of coherent and noncoherent detection. It is shown in [8] that the detector optimally uses the partial channel knowledge to result in a combination of coherent and noncoherent detection with weights determined according to the channel variation rate and the quality of the estimate. As nodes are distributed in a cooperative network, all the channels need not be rapidly varying. In such a case, the performance improvement will be much more. We do not consider differential modulation based cooperation because even differential schemes succumb to an error floor in a rapidly time varying channel [8], [9].

The system model for cooperation with BFSK modulation is given by,

$$
\begin{gathered}
\mathbf{y}(k)=h(k) \mathbf{x}(k)+\mathbf{n}_{D 1}(k) \\
\mathbf{r}(k)=g(k) \mathbf{x}(k)+\mathbf{n}_{R}(k) \\
\mathbf{z}(k)=f(k) \mathbf{x}_{r}(k)+\mathbf{n}_{D 2}(k),
\end{gathered}
$$

where the transmitted and received symbols are vectors with two components representing the two orthogonal bands of BFSK.

$$
\mathbf{x}_{r}(k)=\beta_{k} \mathbf{r}(k)
$$

$\beta_{k}$ is chosen to satisfy an average energy constraint per symbol. For DF systems, the symbol detected at the relay is transmitted with its own power constraint.

$$
\mathbf{x}_{r}(k)=\widehat{\mathbf{x}}_{k} .
$$




\section{A. Demodulate and Forward}

ML detection rule for a single relay demodulate and forward scheme [10] can be generalized as

$$
\hat{d}_{k}=q_{1}+\ln \left(\frac{\frac{\epsilon(k)}{1-\epsilon(k)}+\exp \left(q_{2}\right)}{1+\frac{\epsilon(k)}{1-\epsilon(k)} \exp \left(q_{2}\right)}\right) \lessgtr_{1}^{0} 0,
$$

where $q_{1}, q_{2}$ and $\epsilon$ are obtained according to the detection strategies at the relay and the destination. Detection rule for coherent detection with FSK in a time varying channel can be directly obtained from the result in Section IV. In the rest of the section, we provide ML detection rules for various partially coherent detectors.

1) Partially Coherent Direct link and Non Coherent Decoding at the relay: When it is hard to obtain partial channel knowledge at the relay, noncoherent detection of S-R link. In this detection strategy, the relay performs noncoherent detection and the R-D link is noncoherently detected at the destination. The direct link is detected with the partial channel knowledge and optimal combining results in the following decision rule.

$$
\begin{gathered}
q_{1}=\frac{2 a_{1}^{k} \sqrt{E_{s}} \mathfrak{R e}\left[h_{0}^{*}\left(y_{1}(k)-y_{2}(k)\right)\right]+\left(1-a_{1}^{2 k}\right) E_{s}\left(\left|y_{1}(k)\right|^{2}-\left|y_{2}(k)\right|^{2}\right)}{N_{0}\left(\left(1-a_{1}^{2 k}\right) E_{s}+N_{0}\right)} \\
q_{2}=\frac{\sigma_{f}^{2} E_{s}\left[\left|z_{1}(k)\right|^{2}-\left|z_{2}(k)\right|^{2}\right]}{N_{0}\left(\sigma_{f}^{2} E_{s}+N_{0}\right)} \\
\epsilon(k)=\frac{1}{2+\frac{\sigma_{g}^{2} E_{s}}{N_{0}}}
\end{gathered}
$$

2) Partially Coherent Direct Transmission and Relaying: When partial channel knowledge is utilized at all the nodes, the detection rule in the form (17) is by

$$
\begin{gathered}
q_{1}=\frac{2 a_{1}^{k} \sqrt{E_{s}} \mathfrak{R e}\left[h_{0}^{*}\left(y_{1}(k)-y_{2}(k)\right)\right]+\left(1-a_{1}^{2 k}\right) E_{s}\left(\left|y_{1}(k)\right|^{2}-\left|y_{2}(k)\right|^{2}\right)}{N_{0}\left(\left(1-a_{1}^{2 k}\right) E_{s}+N_{0}\right)} \\
q_{2}=\frac{2 a_{3}^{k} \sqrt{E_{s}} \mathfrak{R e}\left[f_{0}^{*}\left(z_{1}(k)-z_{2}(k)\right)\right]+\left(1-a_{3}^{2 k}\right) \sigma_{f}^{2} E_{s}\left(\left|z_{1}(k)\right|^{2}-\left|z_{2}(k)\right|^{2}\right)}{N_{0}\left(\left(1-a_{3}^{2 k}\right) \sigma_{f}^{2} E_{s}+N_{0}\right)} \\
\epsilon(k)=\frac{1}{2}\left(1-a_{2}^{k} \sqrt{\frac{\sigma_{g}^{2} E_{s}}{\sigma_{g}^{2} E_{s}+2 N_{0}}}\right) .
\end{gathered}
$$

A tight upper bound for partially coherent detection is used to approximate the error probability at the relay, when it resorts to partially coherent detection.

$$
\epsilon(k)=\min \left(\frac{1}{2}\left(\left(1-a_{2}^{k} \sqrt{\frac{\sigma_{g}^{2} E_{s}}{\sigma_{g}^{2} E_{s}+2 N_{0}}}\right), \frac{1}{2+\frac{\sigma_{g}^{2} E_{s}}{N_{0}}}\right)\right.
$$

This is due to the fact that partially coherent detection at any link is superior to both coherent and noncoherent detection for any symbol position $k$.

Fig. 4 compares the performance of the partially coherent detectors with pure coherent and noncoherent detection. It can be seen that the partially coherent detector(detector 2) is superior to the best of coherent and noncoherent detection. A gain of about $2 \mathrm{~dB}$ is achieved over a wide range of SNR. At the crossing of coherent and noncoherent curves, the performance gain is nearly $3 \mathrm{~dB}$. Detector 1 obtains a gain of more than $1 \mathrm{~dB}$ over noncoherent detection. The gain diminishes at high SNR when the channel estimation error dominates over noise.

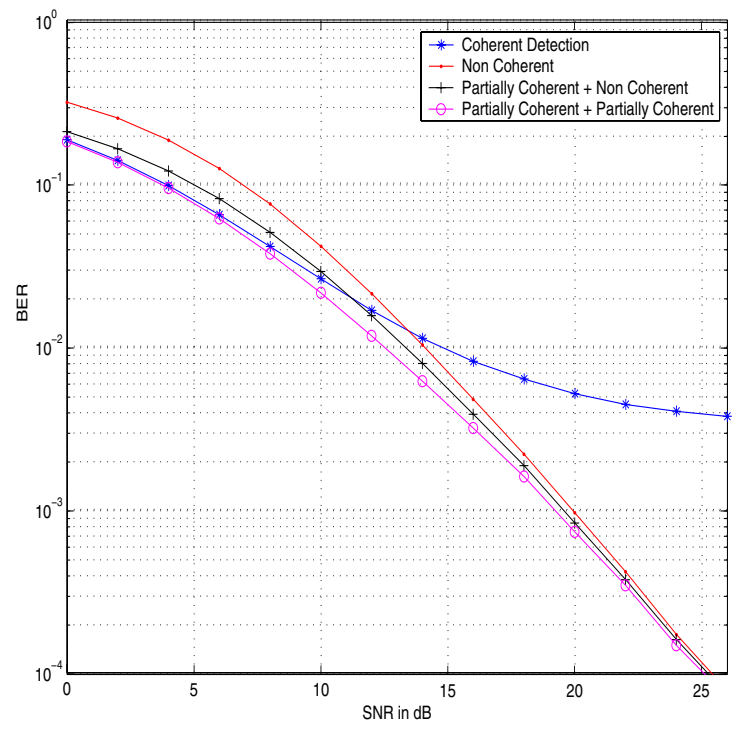

Fig. 4. BER performance of the Demodulate and Forward cooperative schemes for $\left(a_{1}, a_{2}, a_{3}, N\right)=(0.998,0.998,1,50)$.

\section{B. Amplify and Forward}

Partially coherent detection with FSK leads to a simultaneous implementation of both coherent and noncoherent detection followed by an optimal combining [8]. However ML detection rule for noncoherent AF cannot be found in a closed form due to problems indicated in [4], [17]. Thus for AF, we assume the relay-destination link to be non fading $f_{k}=1$, to make the analysis easier as in the case of [4]. It should be noted that both the relay and the destination become static with this assumption and only the source can be mobile. However we provide numerical results for the performance of the detectors for fading R-D channel as well.

1) Partially Coherent Relaying and Direct Transmission: Partially coherent detection is the optimal strategy for any link as the ML rule utilizes the knowledge about the mobility of the nodes. The detector becomes completely coherent when the channel is time invariant or when perfect estimate of the channel is available. Similarly it exhibits noncoherent behavior for the other extreme. Therefore an optimal performance is obtained when both the direct transmission and relayed transmission are detected with partial CSI and the relay employs partially coherent detection. The optimal decision rule is

$$
\begin{gathered}
\widehat{d}_{k}=\frac{\left(1-a_{1}^{2 k}\right) \sigma_{h}^{2}\left[\left|y_{1}(k)\right|^{2}-\left|y_{2}(k)\right|^{2}\right]+2 a_{1}^{k} N_{0} \Re \mathfrak{R}\left[h_{0}^{*}\left(y_{1}(k)-y_{2}(k)\right)\right]}{\left(\left(1-a_{1}^{2 k}\right) \sigma_{h}^{2}+N_{0}\right)}+ \\
\frac{\beta^{2}\left(1-a_{2}^{2 k}\right) \sigma_{g}^{2}\left[\left|z_{1}(k)\right|^{2}-\left|z_{2}(k)\right|^{2}\right]+2 \beta a_{2}^{k}\left(1+\beta^{2}\right) N_{0} \Re \mathfrak{e}\left[g_{0}^{*}\left(z_{1}(k)-z_{2}(k)\right)\right]}{\left(1+\beta^{2}\right)\left[\beta^{2}\left(1-a_{2}^{2 k}\right) \sigma_{g}^{2}+\left(1+\beta^{2}\right) N_{0}\right]} \\
\lessgtr_{1}^{0} 0
\end{gathered}
$$

2) Non Coherent Relaying and Partially Coherent Direct Link: For optimal partially coherent detection of the relayed transmission, the destination must have the knowledge of the $\mathrm{S}-\mathrm{R}$ link, which is very hard to obtain in practice. Thus noncoherent detection of relayed transmission and partially coherent detection of the direct path is an ideal combination. The optimal decision rule is 


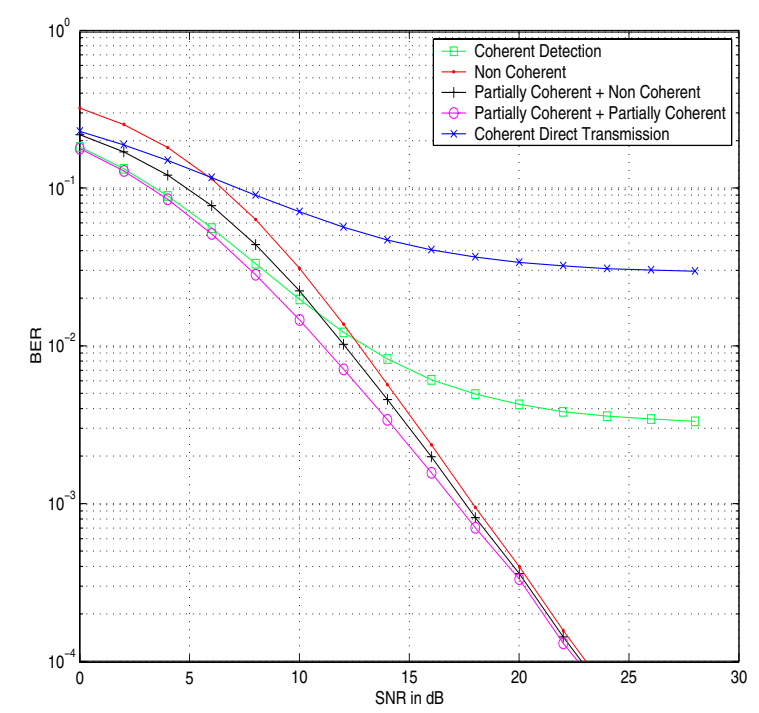

Fig. 5. Amplify and Forward with FSK Modulation, $\left(a_{1}, a_{2}, a_{3}, N\right)=$ $(0.998,0.998,1,50)$, AWGN $R-D$ channel.

$$
\begin{aligned}
& \widehat{d}_{k}=\frac{2 a_{1}^{k} \sqrt{E_{s}} N_{0} \Re \mathfrak{e}\left[h_{0}^{*}\left(y_{1}(k)-y_{2}(k)\right)\right]+\left(1-a_{1}^{2 k}\right) E_{s}\left[\left|y_{1}(k)\right|^{2}-\left|y_{2}(k)\right|^{2}\right]}{\left(1-a_{1}^{2 k}\right) E_{s}+N_{0}} \\
& +\frac{\beta^{2} \sigma_{g}^{2} E_{s}\left[\left|z_{1}(k)\right|^{2}-\left|z_{2}(k)\right|^{2}\right]}{\left(1+\beta^{2}\right)\left(\beta^{2} \sigma_{g}^{2} E_{s}+\left(1+\beta^{2}\right) N_{0}\right)} \lessgtr_{1}^{0} 0
\end{aligned}
$$

Fig. 5 shows the performance of these detectors along with the conventional coherent and noncoherent detectors for an AWGN R-D link. Fig. 6 plots the performance for a fading $\mathrm{R}-\mathrm{D}$ link. It can be noticed that the gain achieved by these detectors is not affected when the relay destination link is a fading channel.

\section{CONCLUSION}

In this work, we considered the problems posed by node mobility in a cooperative network. To study the effect of channel uncertainty caused by mobility, we restricted the channel estimation rate such that the overhead caused by it is minimal. This resulted in severe performance degradation leading to an error floor for both AF and DF systems. We also demonstrated that the impact of mobility on the performance is greatest when the source is mobile, despite the symmetry in the network. We demonstrated the need for more robust modulation schemes like FSK that are not greatly affected by the coherence of the channel estimate. Exploiting this property, we proposed a variety of detection rules, each suitable for a particular mobile scenario. These detectors are hybrids of partially coherent detectors and noncoherent detectors. The performance of these detectors is superior to both coherent and noncoherent detectors in fast fading and a gain of about $2 \mathrm{~dB}$ is obtained over a wide range of SNR. As energy efficiency is one of the main objectives for pursuing cooperation and relaying, these hybrid detectors assume significance in fast fading scenarios.

\section{REFERENCES}

[1] A. Sendonaris, E. Erkip, and B. Aazhang, "User cooperation diversity, Part I: System description," IEEE Transaction On Communications, vol. 51, no. 11, pp. 1927-1938, 2003.

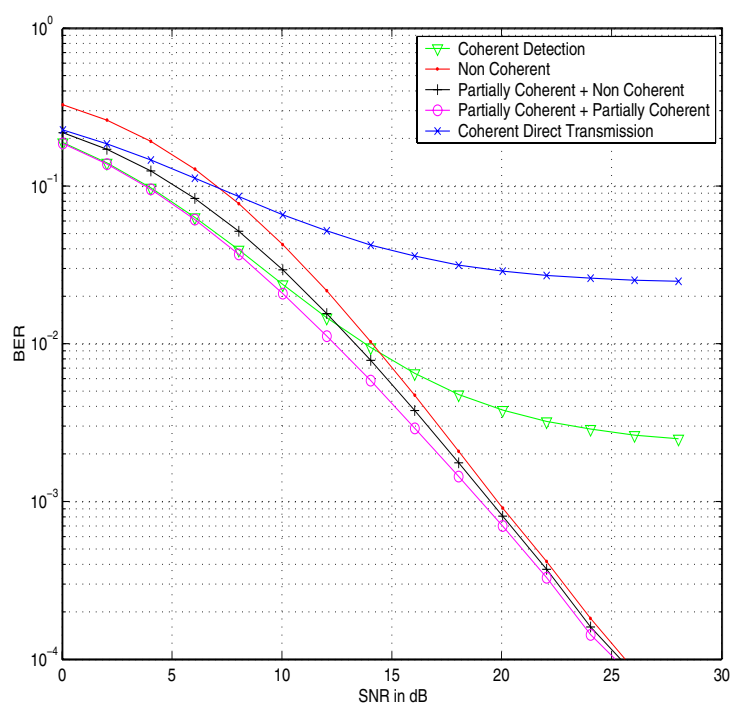

Fig. 6. Amplify and Forward with FSK Modulation, $\left(a_{1}, a_{2}, a_{3}, N\right)=$ $(0.998,0.998,1,50)$, Fading $R-D$ channel.

[2] J. N. Laneman and G. W. Wornell, "Exploiting Distributed Spatial Diversity in Wireless Networks ," in in Proc. Allerton Conf. Commun., Contr., Computing, Illinois, Oct 2000.

[3] A. Ribeiro, X. Cai, and G. B. Giannakis, "Symbol Error Probabilities for General Cooperative Links ," IEEE Transactions on Wireless Communications, vol. 4, no.3, pp. 1264-1273, May 2005.

[4] R. U. Nabar, H. Bölcskei, and F. W. Kneubühler, "Fading relay channels: Performance limits and space-time signal design," IEEE Journal on Selected Areas in Communications, vol. 14, pp. 1099-1109, Aug 2004.

[5] J. N. Laneman and G. W. Wornell, "Distributed Space-Time Coded Protocols for Exploiting Cooperative Diversity in Wireless Networks," in Proc. IEEE Global Comm. Conf. (GLOBECOM), Taipei, Taiwan, Nov 2002.

[6] T. E. Hunter and A. Nosratinia, "Diversity through coded cooperation," IEEE J. Select. Areas Commun., submitted for publication.

[7] J. Boyer, D. D. Falconer, and H. Yanikomeroglu, "A theoretical characterization of the multihop wireless communications channel with diversity," in in Proc. Global Telecommunications Conf., San Antonio, TX, Nov 2001.

[8] K. Gomadam and S. A. Jafar, "Modulation and Detection for simple receivers in rapidly time varying channels " IEEE Transactions on Communications, Submitted for Publication.

[9] K. Gomadam and S. A. Jafar, "Partially Coherent Detection in Rapidly Time Varying Channels.," in Proc. IEEE WCNC, 2006.

[10] D. Chen and J. N. Laneman, "Modulation and demodulation for cooperative diversity in wireless systems," To appear in IEEE Trans. Wireless Commun., 2005.

[11] M. Zorzi, R. R. Rao, L. B. Milstein, "On the accuracy of a first-order Markov model for data transmission on fading channels.," in Proc. IEEE International Conference on Universal Personal Communications. 1995.

[12] W. C. Jakes and D. C. Cox, eds., Microwave Mobile Communications. Wiley-IEEE Press, 1994.

[13] M. O. Hasna and M.-S. Alouini, "A performance study of dual-hop transmissions with fixed gain relays.," in Proc. Vehicular Technology Conf., Birmingham, AL, 2002.

[14] M. K. Simon, S. M. Hinedi and W. C. Lindsey, Digital Communication Techniques, Signal Design and Detection. PTR Prentice Hall.

[15] J. G. Proakis, Digital Communications. New York: McGraw-Hill, 4th ed., 2001.

[16] Z. Wang and G. B. Giannakis, "A simple and general parameterization quantifying performance in fading channels.", IEEE Trans. Commun., vol. 51, no. 8, pp. 1389-1398, 2003.

[17] D. Chen and J. N. Laneman, "Cooperative Diversity for Wireless Fading Channels without Channel State Information," in in Proc. Asilomar Conf. Signals, Systems, and Computers, Monterey, CA, Nov 2004. 